\title{
Recognize Color Face Images Using Complex Eigenfaces
}

\author{
Jian Yang ${ }^{1}$, David Zhang ${ }^{1}$, Yong $\mathrm{Xu}^{2}$, and Jing-yu Yang ${ }^{3}$ \\ ${ }^{1}$ Department of Computing, Hong Kong Polytechnic University, Kowloon, Hong Kong \\ \{csjyang, csdzhang\} @comp.polyu.edu.hk \\ http: / / www4 . comp.polyu.edu.hk/ biometrics / \\ ${ }^{2}$ Bio-Computing Research Center and Shenzhen graduate school, \\ Harbin Institute of Technology, Shenzhen, China \\ laterfall2@yahoo.com.cn \\ ${ }^{3}$ Department of Computer Science, Nanjing University of Science and Technology, \\ Nanjing 210094, P.R. China \\ yangjy@public1.ptt.js.cn
}

\begin{abstract}
A strategy of color image based human face representation is first proposed. Then, based on this representation, complex Eigenfaces technique is developed for facial feature extraction. Finally, we test our idea using the AR face database. The experimental result demonstrates that the proposed color image based complex Eigenfaces method is more robust to illumination variations than the traditional grayscale image based Eigenfaces.
\end{abstract}

\section{Introduction}

In recent years, face recognition has become a very active research area. Up to now, numerous techniques for face representation and recognition have been developed [1]. However, almost all of these methods are based on grayscale (intensity) face images. Even if the color images are available, the usual way is to convert them into grayscale images and then base on them to recognize. Obviously, in the process of image conversion, some useful discriminatory information contained in the face color itself is lost. More specifically, if we characterize a color image using color model such as HSV (or HSI), there are three basic color attributes, i.e., hue, saturation and intensity (value). Converting color images into grayscale ones means that the intensity component is merely employed while the two other components are discarded. Does there exist some discriminatory information in hue and saturation components? If so, how to make use of these discriminatory information for recognition? And, as we know, the intensity component is sensitive to illumination conditions, which leads to the difficulty of recognition based on grayscale images. Now, another issue is: can we combine the color components of image effectively to reduce the disadvantageous effect resulting from different illumination conditions as far as possible?

In this paper, we try to answer these questions. We make use of two color components, saturation and intensity (rather than the single intensity component), and combine them together by a complex matrix to represent face. Then, the classical Eigenfaces $[2,3]$ is generalized for recognition. The experimental result on AR face database demonstrates that the suggested face representation and recognition method outperforms the usual grayscale image based Eigenfaces. 


\section{Face Representation in HSV Color Space}

Since it is generally considered that the HSV model is more similar to human perception of color, this color model is adopted in this paper. The common RGB model can be converted into HSV by the formulations provided in [4]. Fig. 1 shows the three HSV components, i.e., hue, saturation and (intensity) value corresponding to image (a), (b) and (c), respectively. From Fig. 1, it is easy to see that the illumination conditions of image (a), (b) and (c) are different and the component hue is most sensitive to lighting variation. So, we decide to use the saturation and value components to represent face. These two components can be combined together by a complex matrix

$$
\text { Complex-matrix }=\mu_{1} S+i \mu_{2} V
$$

where $i$ is imaginary unit, $\mu_{1}$ and $\mu_{2}$ are called combination parameters.

Note that the parameters $\mu_{1}$ and $\mu_{2}$ are introduced to reduce the effect of illumination variations. Here, we select $\mu_{1}=1 / m_{1}, \mu_{2}=1 / m_{2}$, where, $m_{1}$ is the mean of all elements of component $\mathrm{S}$, and $m_{2}$ is the mean of all elements of component $\mathrm{V}$.

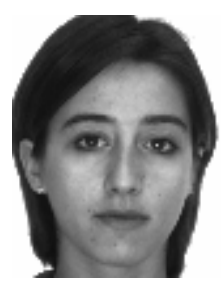

(a)

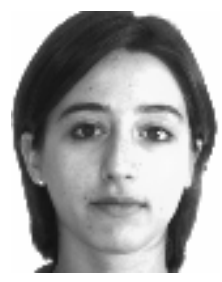

(b)

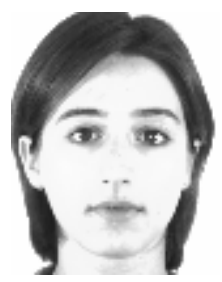

(c)

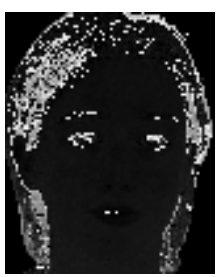

$(\mathrm{a}-\mathrm{H})$

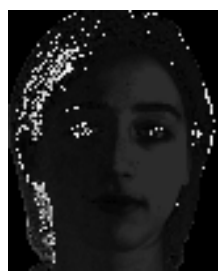

(b-H)

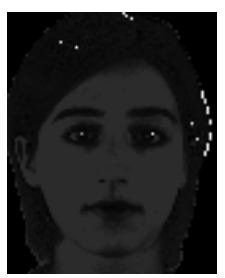

(c-H)

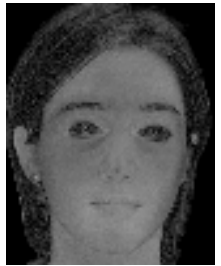

$(\mathrm{a}-\mathrm{S})$

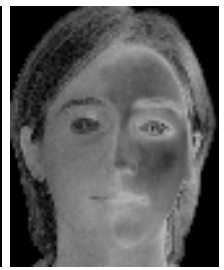

(b-S)

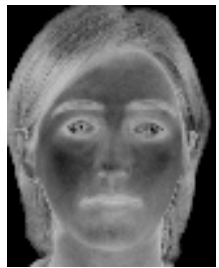

$(\mathrm{c}-\mathrm{S})$

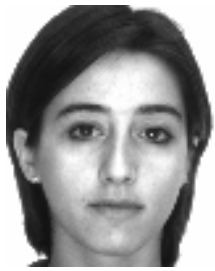

$(\mathrm{a}-\mathrm{V})$

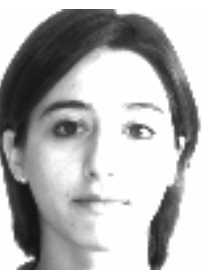

(b-V)

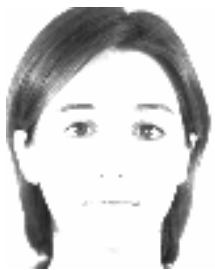

$(\mathrm{c}-\mathrm{V})$

Fig. 1. Three images under different illumination conditions and their corresponding hue $(\mathrm{H})$, saturation (S) and value (V) component images 
The complex-matrix is used to represent color face. It can be converted into same dimensional complex vector $\mathrm{X}$, which is called the image vector.

\section{Complex Eigenfaces Technique}

In documents [5, 6], principle component analysis (PCA) is generalized to suit for feature extraction in complex feature space. In a similar way, the Eigenfaces technique can be generalized.

The total covariance matrix $S_{t}$ in complex image vector space is defined by

$$
S_{t}=\frac{1}{M} \sum_{i=1}^{m}\left(X_{i}-\bar{X}\right)\left(X_{i}-\bar{X}\right)^{H}
$$

where $H$ is the denotation of conjugate transpose; $M$ is the total number of training samples; $\bar{X}$ denotes the mean vector of training samples.

It is easy to know $S_{t}$ is a non-negative definite Hermite matrix.

Since $n$-dimensional image vectors will result in an $n \times n$ covariance matrix $S_{t}$, if the dimension of image vector is very high, it is very difficult to calculate $S_{t}$ 's eigenvectors directly. As we know, in face recognition problems, the total number of training samples $m$ is always much smaller than the dimension of image vector $n$, so, for computational efficiency, we can adopt the following technique to get the $S_{t}$ 's eigenvectors.

Let $Y=\left(X_{1}-\bar{X}, \cdots, X_{m}-\bar{X}\right), \quad Y \in R^{n \times m}$, then $S_{t}$ can also be denoted by $S_{t}=\frac{1}{M} Y Y^{H}$.

Form matrix $R=Y^{H} Y$, which is a $m \times m$ non-negative definite Hermite matrix. Since $R$ 's size is much smaller than that of $S_{t}$, it is much easier to get its eigenvectors. If we work out $R$ 's orthonormal eigenvectors $v_{1}, v_{2}, \cdots, v_{m}$, and suppose the associated eigenvalues satisfy $\lambda_{1} \geq \lambda_{2} \geq \cdots \geq \lambda_{m}$, then, it is easy to prove that the orthonormal eigenvectors of $S_{t}$ corresponding to nonzero eigenvalues are

$$
u_{i}=\frac{1}{\sqrt{\lambda_{i}}} Y v_{i}, i=1, \cdots, r(r \leq m-1)
$$

And, the associated eigenvalues are exactly $\lambda_{i}, i=1, \cdots, r$. The first $d$ eigenvectors (eigenfaces) are selected as projection axes, and the resulting feature vector of sample $X$ can be obtained by the following transformation

$$
Y=\Phi^{H} X, \text { where, } \Phi=\left(u_{1}, \cdots, u_{d}\right)
$$




\section{Experiment}

We intend to test our idea on AR face database, which was created by Aleix Martinez and Robert Benavente in CVC at the U.A.B [7]. This database contains over 4,000 color images corresponding to 126 people's faces (70 men and 56 women). Images feature frontal view faces with different facial expressions, illumination conditions, and occlusions (sun glasses and scarf). The pictures were taken at the CVC under strictly controlled conditions. No restrictions on wear (clothes, glasses, etc.), makeup, hair style, etc. were imposed to participants. Each person participated in two sessions, separated by two weeks (14 days) time. The same pictures were taken in both sessions. Each section contains 13 color images. Some examples are shown in web page (http://rvl1.ecn.purdue.edu/ aleix/aleix_face_DB.html).

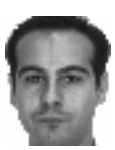

$1-1$

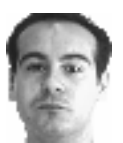

$1-5$

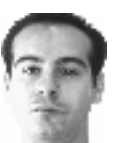

$1-6$

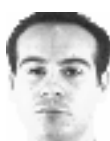

$1-7$

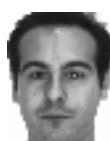

$1-14$

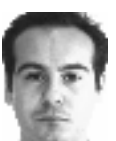

$1-18$

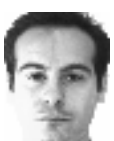

1-19

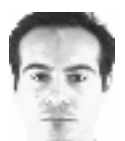

1-20

Fig. 2. The training and testing samples of the first man in the database, where, (1-1) and (1-14) are training samples, the remaining are testing samples

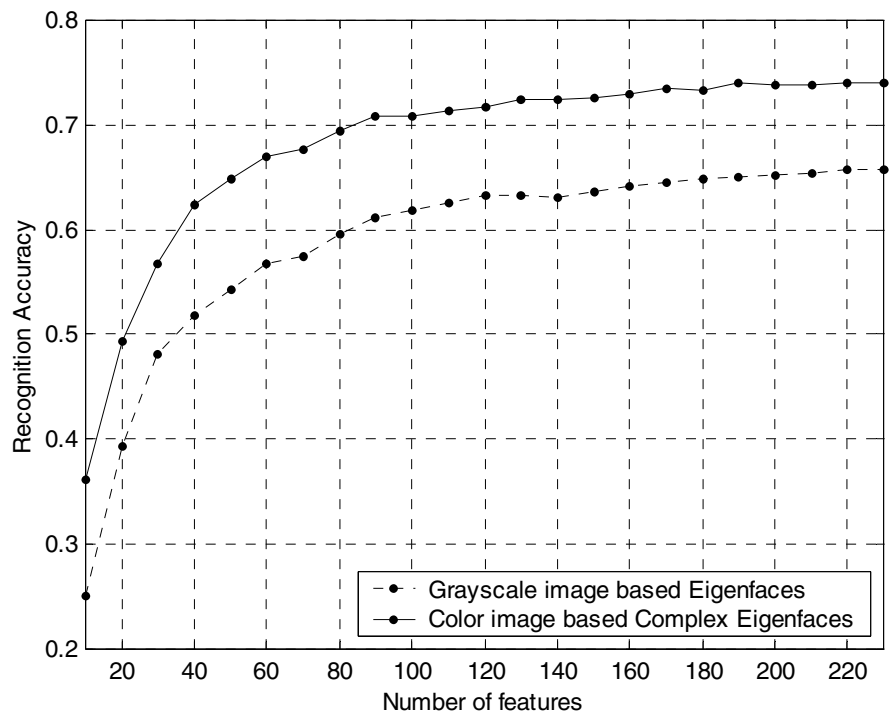

Fig. 3. Comparison of the proposed color image based Complex Eigenfaces and the traditional grayscale image based Eigenfaces under a nearest neighbor classifier (NN)

In this experiment, 120 different individuals (65 men and 55 women) are randomly selected from this database. We manually cut the face portion from the original image and resize it to be $50 \times 40$ pixels. Since the main objective of this experiment is to 
compare the robustness of face representation approaches in variable illumination conditions, we use the first image of each session (No. 1 and No. 14) for training, and the other images (No. 5, 6, 7 and No. 18, 19, 20), which are taken under different illumination conditions and without occlusions, are used for testing. The training and testing samples of the first man in the database are shown in Fig. 2.

The images are first converted from RGB space to HSV space. Then, the saturation (S) and value (V) components of each image are combined together by Eq. (1) to represent face. In the resulting complex image vector space, the developed complex Eigenfaces technique is used for feature extraction. In the final feature space, a nearest neighbor classifier is employed. When the number of selected features varies from 10 to 230 with an interval of ten, the corresponding recognition accuracy is illustrated in Fig. 3.

For comparison, another experiment is performed using the common method. The color images are first converted to gray-level ones by adding the three color channels, i.e., $I=\frac{1}{3}(R+G+B)$. Then based on these grayscale images, classical Eigenfaces $[2,3]$ technique is used for feature extraction and a nearest neighbor classifier is employed for classification. The recognition accuracy is also illustrated in Fig. 3.

From Fig. 3, it is obvious that the proposed color image based complex Eigenfaces is superior to the traditional grayscale image based Eigenfaces. The top recognition accuracy of the complex Eigenfaces reaches $74.0 \%$, which is an increase of $8.3 \%$ compared to the Eigenfaces $(65.7 \%)$. This experimental result also demonstrates that color image based face representation and recognition is more robust to illumination variations.

\section{Conclusion}

In this paper, we first propose a new strategy for representing color face images, that is, to combine the two color attributes, saturation and value, together by a complex matrix. Then, a technique called complex Eigenfaces is developed for feature extraction. The experimental results indicate that the proposed color image based complex Eigenfaces outperforms the traditional grayscale image based Eigenfaces and also demonstrate that the developed color image based face representation and recognition method is more robust to illumination variations.

\section{References}

1. W. Zhao, R. Chellappa, A. Rosenfeld, and P. Phillips, Face recognition: A literature survey. Technical Report CAR-TR-948, UMD CS-TR-4167R, August (2002)

2. M. Turk and A. Pentland, Eigenfaces for recognition. J. Cognitive Neuroscience, 3(1) (1991) 71-86

3. M. Turk and A. Pentland, Face recognition using Eigenfaces. Proc. IEEE Conf. On Computer Vision and Pattern Recognition, (1991) 586-591

4. Y. Wang and B. Yuan, A novel approach for human face detection from color images under complex background. Pattern Recognition, 34 (10) (2001) 1983-1992

5. J. Yang, J.-y. Yang, Generalized K-L transformed based combined feature extraction. Pattern Recognition, 35 (1) (2002) 295-297

6. J. Yang, J.-y. Yang, D. Zhang, J. F. Lu, Feature fusion: parallel strategy vs. serial strategy. Pattern Recognition, 36 (6) (2003) 1369-1381

7. A.M. Martinez and R. Benavente, The AR Face Database. CVC Technical .Report \#24, June (1998) 